Aims. Treatment Escalation Plans (TEP) detail appropriate ceilings of care and guide treatment of patients based on shared decision making. Whilst established in many acute trusts, TEP are not frequently used in community mental health hospitals. This is particularly concerning in organic mental health wards, where patients with severe dementia may be transferred to acute hospitals for treatment without consideration about whether this is appropriate. Our aim for this quality improvement project was to develop and implement TEP within a community mental health hospital to support the management of our older patients with severe mental illness.

Method. We designed a TEP form based on a prototype used in a partner acute trust and evaluated its use on our wards, comprising 20 patients between August-September 2020. This included clear options of the different ceilings of care and what they comprised of for our patients. We obtained quantitative data on the use of TEP, including the length of time from admission to completion, as well as qualitative data from healthcare staff regarding their experience of using TEP.

Result. TEP implementation was feasible and well received among members of staff. All 20 patients had a TEP in place within 2 weeks of admission. The mean number of days taken to complete a TEP form in August-September was 7.1. A snapshot done 2 months later showed new admissions had a mean number of days to complete TEP reduced to 3.2. There was an improvement in understanding the purpose, comprehensiveness and location of TEP forms during their implementation. The key theme that arose from qualitative analysis of healthcare staff comments was that TEP forms provided clear guidance on the appropriateness of escalation of care.

Conclusion. TEP forms offer clear guidance to treating clinicians about the ceilings of care for patients. This also allows for open conversations with patients or their next of kins regarding ceiling of care. This is especially important in mental health inpatients with dementia, when escalation of treatment is not always appropriate. TEP were successfully implemented in our community mental health hospital and we plan further post-implementation evaluation. We intend to roll out the TEP form across our mental health trust and share findings globally to promote best practice.

Driving digitisation of the workforce. Junior doctor's digital devices rollout at South London and Maudsley NHS Foundation Trust: an early review of uptake, usage and effects

\section{Tharun Zacharia}

South London and Maudsley NHS Foundation Trust, IoPPN King's College London

doi: 10.1192/bjo.2021.209
Aims. A Quality Improvement Project (QIP) was completed at South London and Maudsley NHS Foundation Trust (SLaM) to provide access of Trust laptops and smartphones (digital devices) to training doctors.

The aim of the study was to assess the views of trainees before and after the rollout of digital devices. Also, to assess barriers of device uptake/usage.

Method. Trainee doctors were surveyed before mass release of digital devices. Trainees were surveyed again 2 months later, providing ample opportunity to request a device.

Correlated survey questions asked about training grade, expectation to request a digital device (and subsequent completed request), expected usage cases (and actual usage cases), expected benefits (and actual benefits), perceived importance of access to each device (before and after access) and barriers for device uptake. Also inquired were general comments about the project and actual lead time on device access.

Result. 110, mixed training grade, doctors participated in both surveys combined. There was a high demand for digital devices. Laptop requests were highest, with greater clarity of potential usage cases noted for a laptop. Laptops were perceived to be the more important device to a trainee.

Common usage cases for laptops were clinical work (in and out of working hours). There was also high usage around educational and audit/QIP activities. Smartphones were used only for clinical work (in and out of working hours).

Having access to each device was most beneficial in saving clinician time. Other sizable benefits included improvements in communication with patients and other professionals, as well as benefits toward patient safety. The laptop specifically also improved educational access.

A larger proportion of trainees requested a mobile phone than were previously expecting to. New ways of working due to the pandemic were noted to be motivating factors toward usage of devices.

The biggest barrier to device uptake was the delay from request to acquisition (often over 4 weeks).

Numerous respondents used personal devices for Trust related activities. This was more prevalent with smartphones, than with laptops.

Conclusion. Digital device rollout was a valued endeavour, with enhanced demand secondary to the pandemic. Primary usage and benefits supported clinical effectiveness. The primary access barrier was the delay from point of request to point of delivery. As SLaM, and the NHS as a whole, becomes increasingly digitised, this program is vital to allow the Trust to ensure the trainee workforce are digitally equipped to continue to deliver safe, effective and quality care. 